\title{
THALASSEMIA -AN OVERVIEW
}

\section{Genetics}

\section{Muskan Gupta $\quad$ SRM Institute Of Science And Technology, Kattankulathur.}

\section{ABSTRACT}

Thalassemia is a hereditary disorder found on chromosome 11 and chromosome no 16, caused due to point mutation/insertion/deletion. It is one of the most prevalent single gene disorder found in the Indian population with a carrier frequency of 3-4\%. The individuals are categorized into 3 types: homozygous, heterozygous and compound heterozygous. Where heterozygous individuals are normally carriers and homozygous and compound heterozygous are affected. The severity of this disorder lies on what type of mutation the gene carries. Previous studies showed the use of ARMS-PCR for the detection of targeted mutations and other showed the use of Sanger's sequencing for the study of the mutation. Some new or rare mutations can also be detected through Sanger Sequencing and can help in studying the spectrum of the gene. The Clinical treatment involves blood transfusion or allogeneic stem cell transplant. There are lots of complications arising during treatment such as iron overloading. Detection of mutation in the family helps in prenatal testing to prevent the birth of affected children in the family.

\section{KEYWORDS}

Beta-Thalassemia, Alpha-Thalassemia, Genomics, HBB Gene, Mutation

\section{INTRODUCTION}

Thalassemia is a type of hereditary blood disorder that causes ineffective RBC production in the body due to which the patient suffers from moderate to severe anemia. The cause of this type of genetic disease lies on chromosome no 11 and chromosome no 16 where the beta-globin gene known as HBB (hemoglobin beta gene) is present which is responsible for the production of hemoglobin. Hemoglobin is the iron-containing protein in RBCs that conveys oxygen to the cells throughout the body (Galanello, 2010). This is an autosomal recessive disease and the, chances that the offspring will be affected are $25 \%$ when both parents are carrier.

Since thalassemia is caused due to defect in the $\alpha$ and $\beta$ globulin genes it is classified into two types: $\alpha$-thalassemia and $\beta$-thalassemia. $\alpha$ thalassemia is transcribed from four alleles ( $\mathrm{HbA} 1$ and $\mathrm{HbA} 2$ genes) and $\beta$-thalassemia from 2 alleles (HBB)

Apart from this the unpaired polypeptide chain becomes insoluble and tends to precipitate. The precipitation of these unpaired polypeptide chains results to form inclusion bodies which further damages the RBC (Mandal, 2010) (Galanello, 2010)In relationship with this the absence of beta chain synthesis prompts the production of an overabundance of alpha globulin chain and vice-versa. Therefore, The defect in RBC production later gives ascends to what is known as microcytic anemia.

\section{CLASSIFICATION}

\section{Alpha-Thalassemia}

The defect in one or all 4 alleles at chromosome 16 that fail in RBC production (of alpha-globulin gene) causes alpha-thalassemia.

\section{The severity of this disorder is grouped into 4 forms:}

1. Silent carrier: people diagnosed with silent carrier contain one damaged or missing allele out of four $(\alpha \alpha, \alpha)$ ). The size of the $\mathrm{RBC}$ is smaller than usual but no other symptoms may arise.

2. Alpha-thalassemia trait/carrier: Out of the four alleles two of the alleles are damaged or deleted from one chromosome $(\alpha \alpha, 00)$ or each $\left(\alpha_{0}, \alpha_{0}\right)$. The trait suffers from mild anemia

3. Hemoglobin $\mathbf{H}$ : This form result in the deletion of all three alleles leaving only one allele working. Patients with $\mathrm{Hb} \mathrm{H}$ suffer from moderate to severe anemia and sometimes the health can worsen if exposed to certain chemicals, medication, etc.

4. Alpha-thalassemia major: This condition arises when all the 4 alleles are missing $(\mathrm{oo}, \mathrm{oo})$ which leads to severe anemia or hydrops fetalis syndrome. The patient suffers from marked hepatosplenomegaly, skeletal malformation and also can lead to the death of the fetus in utero. (Galanello R, 2011)

\section{Beta-thalassemia}

Only one Beta-globulin gene (HBB) is located on chromosome 11 and is classified into 3 types:

1. Thalassemia minor: Thalassemia minor are usually asymptomatic carriers with low RBC indices. Sometimes they suffer from mild anemia and are represented as $\beta / \beta^{0}$ or $\beta / \beta^{+}$thalassemia.

2. Thalassemia intermedia: Individuals with thalassemia intermedia have one allele absent or have a mutated allele $\beta^{+} / \beta^{0}$ or $\beta^{+} / \beta^{+}$. The severity of the disease lies in different kinds of mutations present. Intermedia patients usually have mild anemia requiring less blood transfusion but in most severe cases the need for blood transfusion may occur throughout life. Symptoms may arise in children between 6 months to 2years, although they are capable of acquiring less blood transfusion but the growth development and mental condition is stagnated.

Clinical complications can arise even-though with the patient having low blood transfusions such as the formation of enucleate $\mathrm{RBC}$ due to an increase in the proliferation of erythroblasts which may fail to differentiate further causing premature death or enucleate RBC production, thereby resulting into anemia (Gupta R, 2018) Other clinical factors such as iron overload, splenectomy, leg ulcer, thrombophilia, infertility. Other; bone deformities lead to osteoporosis, joint, and cardiac bone pain. Heart disease is quite a cause of death.

3. Thalassemia Major: Thalassemia Major also known as Cooley's anemia has deleted/no synthesis of the beta globulin chain $\left(\beta^{0} / \beta^{0}\right)$. Children with thalassemia major can be detected within the first year of birth and it can be possible when both the parents are either carriers or affected. They fail to produce normal adult hemoglobin and usually generate paleness, jaundice, bone deformities on the face (A.D.A.M Inc, 2020) and sometimes fail to thrive during birth. Regular transfusion therapy is involved in less than 2 weeks apart to maintain a $\mathrm{Hb}$ count of $7-10 \mathrm{~g} / \mathrm{dl}$, sometimes there are adverse reactions during the transfusion therapy which exposes the patient to allergic reactions, acute hemolytic reactions, delayed transfusion reactions (Cappellini MD, 2008). Other complications include iron overloading in children which deteriorate their mental and physical conditions and also delay sexual maturation (Galanello, 2010) Thalassemia major if left untreated can lead to heart failure and other liver-related problems.

4. Dominant $\beta$-thalassemia: It is an exceptionally rare condition in which the person having only one mutated allele shows the same symptoms with that of thalassemia major. The affected individual suffers from mild to severe anemia, jaundice, or splenomegaly (NORD)

\section{Prevalancy of Thalassemia}

The global distribution of this hemoglobin disorder according to WHO shows that every year over 50,000 children are born with severe form of thalassemia and around $80 \%$ of them are born in developing countries (Kantharaj, 2018)(WHO, 2008) .Although the inheritance of the gene comes from both the parents there is no inclination in the gender. Approximately, $5 \%$ of the whole world population carry one 
variation in alpha or beta gene of the hemoglobin molecule but by this, we can't say that all of them are symptomatic but can also be carrier/trait. Out of this $5 \%$, only $1.7 \%$ are diagnosed with thalassemia trait. (WHO, 2008) The prevalancy of $\alpha$-thalassemia trait is most commonly found in Sub-Saharan African region with $50 \%$ of carriers and the Western Pacific region with $60 \%$ of carriers. Other geographical regions include America, Europe, the EastMediterranean region, and Southeast-Asia.

For $\beta$-thalassemia, the most common prevalent regions are the EastMediterranean region with $2-18 \%$ of the affected gene population and Southeast-Asia with $0-11 \%$ of the affected gene. Both alpha and betathalassemia mostly occur in tropical and subtropical regions due to the warmer temperature or where malaria has been epidemic. The reason is still unknown. (NEWS-MEDICAL.NET, 2018)

\section{Associated Hemoglobin abnormalities}

$\beta$-thalassemia also occurs in association with other abnormalities in the hemoglobin such as $\mathrm{HbE} / \beta$-thalassemia, $\mathrm{HbC} / \beta$-thalassemia, and $\mathrm{HbS} / \beta$-thalassemia. $\mathrm{HbE} / \beta$-thalassemia patients suffer from very mild anemia which can be compensated by a correct shift in the oxygen dissociation curve (Thein.S.L, 2017) .One of the clinical features involving $\mathrm{HbE} / \beta$-thalassemia is the spectrum where at one end there is highly similar to that of severe thalassemia major and another end the patient develops and grows without any need for blood transfusions. The symptoms doesn't show for children until the age of 6 because they remain asymptomatic with a high level of $\mathrm{HbF}$ but as $\mathrm{HbF}$ decreases and is replaced by $\mathrm{HbE}$, symptoms such as anemia and splenomegaly generates. All molecular basis of $\alpha / \beta$ chain in association with $\mathrm{HbE}$ is discussed by(Thein, 2013)

\section{HbH disease (Hemoglobin $\mathrm{H}$ )}

The condition of $\mathrm{HbH}$ varies from person to person. The $\mathrm{HbH}$ disease can be caused due to two factors: Deletional $\mathrm{HbH}$ and Non-deletional $\mathrm{HbH}$ in combination or alone. Deletional $\mathrm{HbH}$ is caused when the combination of deleted mutations remove 3 out $4 \alpha \alpha$ alleles that are responsible for $\alpha$-globulin synthesis. Non-deletional occurs when the combination of deleted mutations remove 2 out of 4 alpha alleles with the removal of one non-deletional point mutation that inactivates the third gene. Out of these two factors, Non-deletional $\mathrm{HbH}$ plays a vital role in causing severe anemia and splenomegaly. (NORD)

\section{RBC Indices}

The hemoglobin count for people with thalassemia major is very less $<7 \mathrm{~g} / \mathrm{dl}$ with $\mathrm{MCV}$ (mean corpuscular volume) of $>50-<70 \mathrm{FL}$ and $\mathrm{MCH}($ mean cell hemoglobin ) of 12-20 pg compared to an average normal human, people with thalassemia major usually don't have a high life expectancy. The other form of milder disease thalassemia intermedia has a hemoglobin count of 7-10 g/dl, MCV of 50-30 fl, and $\mathrm{MCH}$ of $16-24 \mathrm{pg}$. For people with thalassemia intermedia, the condition is severe to less anemia.

\section{Genomics of Thalassemia}

Thalassemia affects both males and females so we cannot say it's gender-based. The alpha and beta-globulin (HBB) is transferred from parents to the offspring. The probability is 1 out of $4(25 \%)$ that the child will be affected. Since the offspring derives one copy of the gene from each parent when both parents are carriers and thalassemia is an autosomal recessive disorder, the child to be a carrier is $50 \%$ the rest $25 \%$ is considered to be normal. (Xuan Shang, 2017).The normal allele variant of HBB gene is $1600 \mathrm{bp}$ long or $1.6 \mathrm{~kb}$ consisting of 3 exons with 5'promoter region and 3' UTR (untranslated region). The gene also contains intron regions in which mutations occur, disrupting the splice sites (Cao, 2010). The inherited genetic analysis of hemoglobinopathies that includes thalassemia and sickle cell has a very diverse nature not only the mutations can be detected by various PCR procedure but also by reverse dot plot, MLPA(Multiple ligation probe amplification), Sanger's Sequencing, gene array system. (Old J, 2012).Till now approximately 200 mutations have been registered all over the world. Some of the common mutations that are frequently found in the Indian population are:

Fig 1.

\begin{tabular}{|l|l|l|}
\hline Mutation & Exon & severity \\
\hline c. $92+5 \mathrm{G}>\mathrm{C}$ & Exon1(Intron) & $\beta+$ \\
\hline c. $47 \mathrm{G}>\mathrm{A}$ & Exon1 & $\beta+$ \\
\hline c. $126 \_129 \mathrm{del}$ CTTT & Exon 2 & $\beta+$ \\
\hline c. $92+1 \mathrm{G}>\mathrm{T}$ & Exon 1 (Intron 1$)$ & $\beta+$ \\
\hline$-619 \mathrm{bp}$ & Exon 3 & $\beta 0$ \\
\hline
\end{tabular}

$\beta^{+}:$reduced or lack of beta-globin synthesis

$\beta^{0}$ : lack of beta-globin synthesis

In the Indian population $-619 \mathrm{bp}$ del is the only common deletion shown in the HBB gene, insertion and deletion is uncommon and not found frequently. IVS (intervening sequence) c. $92+5 \mathrm{G}>\mathrm{C}, \mathrm{c} .92+1$ $\mathrm{G}>\mathrm{T}, \mathrm{c} .92+1 \mathrm{G}>\mathrm{C}$ at the intronic splice sites. From the outset the situation of the transformation was accounted for an IVS 1-5 G>C which implies that there is point change at the fifth position of intron 1 , this classification is as yet accessible in the old diaries and records for the better understanding for the physician and researchers (Mandal, 2010).

\section{Treatment}

Treatment for thalassemia is now easily available, with development in medical science, patients with thalassemia can be treated by Blood transfusions, stem cell transplantation, medications. Blood transfusion depends upon the severity of the disease. Severe the disease less hemoglobin production in the body. By giving blood transfusions means more $\mathrm{RBC}$ will be there to carry out oxygen transportation. Before transfusion, there are certain guidelines by WHO (World Health Organization), the European Union (EU), and the American Association of Blood Bank (AABB) which need to be followed (Cappellini, (2014)) Transfusions can only be given under criteria such as $>7 \mathrm{~g} / \mathrm{dl} \mathrm{Hb}$ with fractures, poor growth, or facial changes. Complication arises when regular blood transfusions may lead to building up of iron (Yawn, 2018) Our blood contains iron $\left(\mathrm{Fe}^{2+}\right)$ which keeps on piling up in the organs like liver, heart, brain and can damage their functioning. To reduce this iron loading medication such a folic acid is given for chelating the iron.

Another complication arises in $10-20 \%$ of patients that is "Alloimmunisation". It can happen to children between 1-3 year of age. Where the transfused blood acts as a foreign body to the immune system and antibodies are produced.(Pierelli, (2014) which destroys the transfused blood.

\section{Bone Marrow Transplant}

BMT is the only definitive cure for the treatment of thalassemia major. Allogenic stem cell transplantation of the Bone marrow can deliver positive results. BMT has the highest survival rate of up to $90 \%$ when done from an HLA-identical sibling. Although the huge disadvantage lies in the fact that most of the affected individuals lack HLA-matched Bone marrow. Bone marrow transplant from related donors has been successful (Gaziev, 2013). If BMT is successful the iron overloading can be reduced by repeated phlebotomy thus decreasing the need for iron chelation. (Galanello, 2010)

\section{Genetic counselling and prenatal testing}

Genetic counseling is advised for all the couples who are diagnosed with thalassemia after prenatal testing (Rosatelli, (2009)Family of Consanguineous marriage has a higher risk of an affected child, in this case, parents are advised for prenatal testing every time before pregnancy. When both parents are carriers, the risk accounts for $25 \%$ that the child is affected, CVS is used to detect the status of the child. 11 weeks by chorionic villus sampling (CVS) or at 16 weeks by taking out amniotic fluid. While for children; blood sample is used for the detection of the mutation. Carrier testing done by determining the RBC indices and $\mathrm{Hb}$ variant by HPLC can detect the chances in a family with high prevalence of thalassemia. With affected children of homozygous or compound heterozygous, prenatal testing is carried out for future pregnancy. Pedigree analysis and psychological counseling is advised for parents.(Mandal, 2010)

\section{Future Aspects}

Investigation for new therapeutic approaches for treatment of thalassemia is still underprocess. The possibility of correction of the molecular defect in hematopoietic stem cells by transfer of a normal gene via a suitable vector or by homologous recombination is being actively investigated (Sadelain, 2007). The most promising results have been shown in mice by using lentiviral vectors(Loewen N.)

\section{REFERENCES}

A.D.A.M Inc. (2020,2/06).MEDLINEPLUS. Retrieved fromNationalInstituteofHealth https://medlineplus gov/ency/article/000587.htm

G. (2010). Beta-thalassemia. Genetics in Medicine, 12, 61-76

Cappellini MD, C. A. (2008). Blood Transfusion Therapy in $\beta$-Thalassaemia Major. In C. A. Cappellini MD, Guidelines for the Clinical Management of Thalassaemi [Internet] (2nd Revised edition ed.). Nicosia (CY): Thalassaemia International 
Federation

4. Cappellini,M.D.((2014)).Guidelinesforthe Management of Transfusion Dependent Thalassaemia (TDT) ((3rded). Nicosia (CY): Thalassaemia International Federation.

5. GalanelloR, C. A. (2011,Feb). Gene test review.Alpha-thalassemia. Genetics in Medicine : Official Journal of the American College of Medical Genetics., 13(2), 83-88.

. Galanello,R.O.(2010,/5/21).Beta-thalassemia. Orphanet Journal of Rare Diseases, 5

7. Gaziev,J.\&.(2013,08/20). Bone marrow transplantation for thalassemia from alternative related donors: Improved outcomes with a new approach. Blood, 122.

8. GuptaR,M.K.(2018,April).Ineffective Erythropoiesis:Anemia and Iron Overload. Hematol Oncol Clin North Am, 32(2), 213-221

9. Kantharaj, A. \&. (2018, 0101$)$. Coping with the burden of thalassemia: Aiming for a thalassemia free world. Global Journal of Transfusion Medicine, 3 .

10. Loewen N., P. E. (n.d.). Lentiviral Vectors. In Z. W. Schaffer D.V. (Ed.), Gene Therapy and Gene Delivery Systems:AdvancesinBiochemicalEngineering/Biotechnology (Vol 99, pp. 169-191). Berlin, Heidelberg.: Springer.

11. Mandal, k. (2010). Genetics in tropics. In P. Gupta (Ed.), Tropical pediatrics (p. 3). Lucknow.

12. NEWS-MEDICAL.NET. (2018). Thalassemia Prevalence.

13. NORD. (n.d.). RARE DISEASE INFORMATION. Retrieved from National Organization for Rare Disorders (NORD): https://rarediseases.org/rare-diseases/ thalassemia-major/

14. Old J, H. C.-S. (2012). HAEMOGLOBIN PATTERN ANALYSIS. In Prevention of Thalassaemias and Other Haemoglobin Disorders (2nd edition ed., Vol. Volume 2). Thalassaemias and Oner Hach

15. Pierelli,A.M.((2014). Red blood cell alloimmunization in sickle cell disease and in thalassaemiacurrent status, future perspectives and potential role of molecular typing. current status, future perspectives and potential role of molecular typing, 106, 197-208.

6. Rosatelli, M. \&. ((2009), 11 15). Prenatal Diagnosis of $\beta$-Thalassemias and Hemoglobinopathies. Mediterranean journal of hematology and infectious diseases., 1 .

17. Sadelain, M. \&. $(2007,02$ 01). Therapeutic Options for Patients with Severe $\beta$ Thalassemia: The Need for Globin Gene Therapy. Human gene therapy, 18, 1-9.

18. Thein, S. L. (2013). The Molecular Basis of $\beta$-Thalassemia. Cold Spring Harbour Perspectives in Mediicine.

19. Thein S.L (2017). Genetic Basis and Genetic Modifiers of $\beta$-Thalassemia and Sickle Cell Disease. In T. J. Malik P., Gene and Cell Therapies for Beta-Globinopathies. Advances in Experimental Medicine and Biology (Vol. 1013). New York (NY): Springer

20. WHO. (2008). Global epidemiology of haemoglobin disorders and derived service indicators.

21. Xuan Shang, X. X. (2017, February). Update in the genetics of thalassemia: What clinicians need to know. Best Practice \& Research Clinical Obstetrics \& Gynaecology, $39,3-15$

22. Yawn, D. H. (2018, June 29). Blood transfusion. Transfusion Procedures And Blood Storage. 